The Official Journal of the Kettil Bruun Society for Social and Epidemiological Research on Alcohol

\title{
Combining visual methods with focus groups: An innovative approach for capturing the multifaceted and complex work experiences of Fetal Alcohol Spectrum Disorder prevention specialists
}

\author{
Jenelle M. Job ${ }^{1}$, Cheryl-Anne Poth ${ }^{2}$, Jacqueline Pei ${ }^{1}$, Katherine Wyper ${ }^{1}$, Teresa O’Riordan ${ }^{3}$, and Lin Taylor ${ }^{4}$ \\ ${ }^{1}$ School \& Clinical Child Psychology, Department of Educational Psychology, University of Alberta \\ ${ }^{2}$ Measurement, Evaluation, \& Cognition, Department of Educational Psychology, University of Alberta \\ ${ }^{3}$ NWC Alberta FASD Services Network \\ ${ }^{4}$ Heart Sights Consulting
}

\begin{abstract}
Aims: This paper discusses the untapped potential of an innovative methodological approach for capturing the experiences of prevention specialists working with women at risk of drug and/or alcohol-exposed pregnancies and live births. Allowing frontline workers to express their personal and professional experiences through a creative activity enhanced our understanding of the difficult-to-measure programming outcomes of a provincial Fetal Alcohol Spectrum Disorder (FASD) strategy.
\end{abstract}

Design: A multi-method design integrating focus groups with a quilting activity.

Setting: Annual General Meeting of the Parent and Child Assistance Program (PCAP)—a prevention program for women at risk for drug and/or alcohol-exposed pregnancies and live births.

Participants: Forty-seven FASD prevention specialists from across Alberta, Canada.

Measurements: Triangulation across data sources: seven focus groups and 49 quilting pieces.

Results: Thematic analysis revealed four themes: change as a process, client-advocate connection, ecological understanding, and evidence-based practice. The findings from this study contribute improved understanding about the significance of relationships, reflection, and research in the work of FASD prevention specialists.

Conclusions: A multi-methods approach (focus groups with a quilting activity) provided an appropriate and trustworthy means of accessing the prevention specialists' programmatic experience; that experience has the strong potential for informing future FASD policy, strategic planning, and programming.

The use of visual methods for documenting and representing the social world has resulted in improved understanding of participant experiences and relationships in research (Banks, 2001; Pink, 2007; Rose, 2001). One such visual method is quilting -an innovative technique that allows the researcher to integrate participants' stories into a meaningful collective experience (Koelsch, 2008). With the aim of gaining an understanding of the experiences of frontline workers involved in the prevention of Fetal Alcohol Spectrum Disorder (FASD), this paper discusses the untapped potential of this methodological approach for capturing the difficult-to-measure programming outcomes of a provincial FASD strategy. The approach was especially relevant for the current population of service delivery personnel involved in the Parent and Child Assistance Program (PCAP), a program dedicated to the support and treatment of women engaged in alcohol and/or drug use, because staff had frequently been recruited for research and program reviews where the process was onerous and the results were of limited use for frontline workers. As such, it was beneficial to include an appropriate and trustworthy new methodology to address specific research questions about specialists' planning, collaboration, training, and service delivery, and to gain insight into how participants' work affects them on a personal level. This inquiry into professional and personal experiences through a multi-method design lends itself well to the relational component of the PCAP model and provides a new tool for the triangulation of data sources, allowing for comprehensive and shared understandings of best practices in FASD planning, service delivery, and client care.

Correspondence: Jenelle M. Job, 6-102 Education North, Department of Educational Psychology, University of Alberta, Edmonton, Alberta, Canada, T6G 2 G5. Telephone: (780) 492-5245, Fax: (780) 492-1318, E-mail: job@ualberta.ca

Financial support: Government of Alberta, Fetal Alcohol Spectrum Disorders Cross-Ministry Committee

Keywords: Fetal alcohol spectrum disorders; multi-methods; focus groups; quilting; prevention 


\section{Prevention: The Why of FASD}

FASD is the leading cause of preventable neurodevelopmental disability in North America, with a conservative incidence rate of approximately nine out of every 1,000 live births (Sampson et al., 1997). FASD results from maternal alcohol consumption during pregnancy and has implications for the child, family, and community, as its features include lifelong deficits in a myriad of domains (Chudley et al., 2005; Streissguth, Sampson et al., 1994), including cognition, executive control, adaptive and social functioning, memory, language, motor coordination, and mental health (Burd, Klug, Martsolf, \& Kerbeshian, 2003; Connor \& Streissguth, 1996; Kelly, Day, \& Streissguth, 2000; Pei, Denys, Hughes, \& Rasmussen, 2011; Rasmussen, 2005). Given its breadth of deficits and diversity of impact, FASD is increasingly recognized in Alberta as a serious and complex issue warranting targeted attention in all government service domains-including healthcare, education, and justice-to ensure appropriate understanding of the condition and support for those affected.

A lack of comprehensive supports for individuals with FASD leads many to experience secondary disabilities, including homelessness, unemployment, criminal involvement, mental health problems, addictions, confinement (psychiatric and correctional), school dropout, sexual deviancy, and problems with parenting (Steinhausen \& Spohr, 1998; Streissguth, Barr, Kogan, \& Bookstein, 1996). The possibility of such adverse outcomes necessitates a clear and delineated system of support, which can only come from an understanding of why services are required and how the needs of individuals with FASDs and their families can best be addressed (Poth \& Pei, 2012).

Recognizing that FASD is preventable must come with the understanding that it is a multifaceted and complicated social and public health concern. For example, the risk of alcohol and/or drug use during pregnancy may be compounded by several factors, including an adverse personal or family history of substance use, poverty, depression, FASD diagnosis, relationship difficulties, and living in a culture tolerant of heavy drinking (Leonardson \& Loudenburg, 2003). With such a range of issues intensifying the challenges faced by women at risk, effective prevention efforts need to be directed toward the coordination of supports and services across domains. It is in this way that the answer to why (prevention) shapes the pathway for how (service delivery), encompassing system collaboration, relationships, and evidence-based practice (Poth \& Pei, 2012). But how do we go about collecting this information effectively and comprehensively?

\section{Visual Methods: The Untapped Potential}

A long-disputed concern within qualitative research is how best to represent participant experiences accurately (Galman, 2009). The magic of an image is its ability to convey multiple messages, provoke questions, and express thoughts and emotions in ways words never could. The multiplicity of messages in visual data makes it useful for the efficient communication of academic knowledge (Weber, 2008). What is emerging is the idea that information may need to be shown as well as stated, so as to reveal elusive, ineffable aspects of knowledge and experience that might otherwise remain hidden. Further, visual mediums allow access to perspectives without the interpretation involved in reading; we can "see someone else's point of view and borrow their experience for a moment" (Weber, 2008, p. 45). As a result, empathetic understanding in viewers is enhanced when they are shown visual stories that compare with their own experiences. Seeing or feeling the details of a participant's lived experience through images, shapes, and colors commonly associated with certain sentiments or events increases the trustworthiness and validity of visual information (Weber, 2008). Similarly, Eisner writes, “. . . artistically crafted work . . creates a paradox of revealing what is universal by examining what is particular" (1995, p. 3).

The inclusion of visual methods in qualitative research permits the acknowledgement that learning is not solely intellectual, but that all senses provide information to which we respond, and researchers are not excluded from this experience (Emmel \& Clark, 2011; Weber, 2008). These methods therefore afford greater flexibility than more traditional approaches (e.g., surveys and interviews) to researchers working in a range of contexts and populations. In the present study, visual approaches enabled us to honor the person behind the information, which in this context was especially significant. As researchers, we agree with Eiser (1995) that the time has come to expand our notion of what constitutes trustworthiness within our research designs, as well as our idea of how academic knowledge can be conveyed and understood.

\section{Study Context}

Background. Many FASD-related programs and services are in their infancy in both development and scope, as basic scientific knowledge and other building blocks for highly effective FASD supports need to be further developed (Gould, Job, St. Arnault, Pei, \& Poth, 2012). To address this, the Government of Alberta (2008) established the FASD Cross-Ministry Committee (FASD-CMC), a partnership of government ministries and provincial and community organizations, for the coordinated development of a unique framework for addressing FASD - the FASD 10-Year Strategic Plan. This made-in-Alberta plan focuses on developing and providing community-based solutions, making it easier for those affected by FASD to receive needed support and resources (Health Council of Canada, 2011). The present study is part of a larger research project designed to evaluate the progress of FASD policy, strategic planning, diagnostic services, and programs during the fifth year of the plan. Areas of research and evaluation include awareness and prevention, support for individuals and caregivers, stakeholder engagement, and training and education (Health Council of Canada, 2011).

The Parent and Child Assistance Program (PCAP). The study participants are FASD prevention specialists involved in PCAP across Alberta. The program is designed to prevent drug and/or alcohol-exposed live births among 
high-risk mothers (Grant, Streissguth \& Ernst, 2002) who have substance abuse issues and are disconnected from community services; these women are exceptionally vulnerable and are in dire need of support and resources (Ernst, Grant, Streissguth, \& Sampson, 1999). The foci on reducing risk and emphasizing the client-advocate relationship are features which distinguish the PCAP model from interventions that force women to remain completely abstinent from drugs and/or alcohol (Ernst et al., 1999).

Upon enrollment in PCAP, clients are matched with highly trained and supervised paraprofessional advocates who share a common history with these women. Clients are enrolled in the program for three years; support is provided through their relationships with the advocates, who complete home visits, offer guidance in effective family planning, give support in attending drug and alcohol treatment, and help in connecting clients with appropriate community services (Grant, Ernst, \& Streissguth, 1999). Providing children with a safe home environment and consistent healthcare is of the utmost importance (Grant, Ernst, \& Streissguth, 1996). To facilitate client success, PCAP specialists are guided by several key objectives, which include establishing a solid client-advocate relationship, developing client-directed goals, and linking to quality community service providers; role modeling and teaching basic skills; and evaluating the client's progress (Grant, Ernst, Streissguth, Phipps, \& Gendler, 1996).

The Alberta PCAP approach is modeled after the Seattle Advocacy Model, which has demonstrated remarkable results. Between program exit and follow-up ( $m=2.5$ years), Grant and colleagues (2003) noted the following successes: an increase in abstinence for six months or more ( $0 \%$ at enrollment to $31 \%$ at exit), a decrease in subsequent pregnancies and alcohol-exposed live births, an increase in permanent housing, and a decrease in public assistance as a main source of income ( $87 \%$ at enrollment to $51 \%$ at exit).

Our research begins to address the need for in-depth understanding of the programmatic experiences of Albertabased FASD specialists who work with women engaged in high-risk behavior to prevent future births of children prenatally exposed to drugs and/or alcohol. This study forwards an innovative methodological approach, integrating focus groups and a quilting activity, in order to address the following research questions: (1) How do FASD specialists work collaboratively in the planning and delivery of related provincial government programs and services? and (2) How are research and best practices used to inform decision-making and practice?

\section{Methods}

A primary goal of the multi-method design integrating focus groups with a quilting activity was to capture the programmatic experiences of FASD prevention specialists from across Alberta, Canada, using an innovative methodological approach that was both appropriate and trustworthy. The methodology was appropriate in that it took into consideration the diversity of client and staff factors, and the emphasis on human connection that is so valued in work with individuals with FASDs and women at risk of drug- and/or alcohol-exposed live births. It was trustworthy in that it met the standards associated with qualitative data (Creswell, 2008). The combination of focus groups and visual methods enabled each participant to share his or her story through conversation and creative expression (quilting). The novel inclusion of a quilting exercise came about through a discussion between the director of PCAP and her friend, a psychologist and quilter with 15 years of experience, about better ways to capture the lived experience of FASD frontline workers and families affected by the disorder. When this conversation was shared with researchers, they suggested that incorporating a quilting activity into the present study would allow for improved ecological understanding and provide new information about the value of visual methods in FASD research. Thus, two data sources were integrated (i.e., focus groups were paired with a visual method, specifically a quilting activity) to enhance methodological rigor and address the notion that reality (i.e., experience) cannot be fully represented through one medium (Emmel \& Clark, 2011).

Study participants were 47 FASD prevention specialists (45 female, 2 male) from across Alberta who were involved in PCAP service delivery and were attending the Alberta PCAP Council Annual General meeting in March 2012. Included in this group were service providers, network coordinators, program managers, administrative personnel, and leadership team members. This diversity of roles and representation from all 10 Alberta networks gave researchers confidence that this sample was demonstrative of the PCAP population and that the quilting pieces produced reflected a range of experiences, emotions, and clientele. All 47 participated in the quilting activity; however, only 41 agreed to also participate in one of seven hour-long focus groups. The groups were organized by role to facilitate open and honest conversation (see Figure 1). Four discussions were held in the first hour, while the remaining participants completed the quilting exercise. The participants then switched activities to ensure that each one had the opportunity to be involved in both data collection phases. Consent for the quilting activity was implied through completion of a piece; participants provided written consent for the focus group portion of the study.

The quilting activity involved participants using a variety of materials (e.g., fabric, markers, glitter glue) to create quilting pieces that expressed their experiences working in PCAP through illustration and/or words. Although a facilitator was present to guide the process, directions were purposefully vague (i.e., "Using words or illustrations, tell us about your experiences in PCAP”). An example of a completed quilting piece was available for viewing, but this piece was unrelated to the topic and was displayed mainly to demonstrate how to use fabric markers and gel paint, and how to prepare a piece that would work as part of an assembled quilt (e.g., thick gel paint lines or excessive coloring may bleed; a one-inch space around illustrations was necessary to ensure they would not be cut off in the sewing process). On the backs of the squares, participants 
included their name, role, and explanation of their piece. As documentation, photographs of the quilting activity and quilt construction were taken, and relevant comments from participants (e.g., feedback about quilting) and our master quilter (e.g., her process of analysis) were noted. For the focus group sessions, a team of experienced researchers used a semi-structured protocol to capture participant experiences, using such guiding questions as “Tell us your story about how the PCAP program has changed over the past five years." The audio recordings of these sessions were transcribed verbatim, and member checking was undertaken through the distribution of summaries generated during a preliminary analysis and solicitation of participant feedback, which was then integrated to ensure data accuracy and response completeness.

Data analysis involved two sequential phases: within individual data source (i.e., within and across focus groups and photographs) followed by integration across data sources (see Figure 2). The individual and cross-focus group analysis followed an inductive and iterative process undertaken independently by two researchers, meaning that coding was generated through transcript readings but code definitions and applications were informed by previous and subsequent transcript readings. Similarities and differences between codes and definitions for each transcript were compared, and an inter-rater reliability of $90 \%$ was achieved and code lists finalized. Categories were generated from tracking tables using the codes relating to service deliverers' experiences engaging in collaborative practice and evidence-based decision-making across focus groups. A subsequent review of transcripts and code lists by a third researcher enhanced confirmability (i.e., the degree to which the themes were grounded in the data) and dependability of themes (Lincoln \& Guba, 1985). The master quilter and a researcher individually undertook the content analysis of the participants' written explanation of the quilting activity, including frequency counts (e.g., shapes) and inductive generation of categories (Galman, 2009). Specifically, attention was paid to common words, images and colors across the pieces, resulting in a hierarchy of categories beginning with pain and grief and ending with hope and vision (see Figure 3). Additionally, researchers engaged in continual examination of the quilting pieces and focus group data for similarities and patterns (Ausband, 2006; Savenye \& Robinson, 2003). The integration of data sources was guided by a constantcomparison approach, whereby categories from the quilting activity were compared for both congruence and dissonance with those from the focus groups. Consensus for final themes and subthemes was achieved through discussion amongst the coders, reviewers, and two principal investigators.

\section{Figure 1}

\section{Summary of data collection of quilting activity and focus groups}
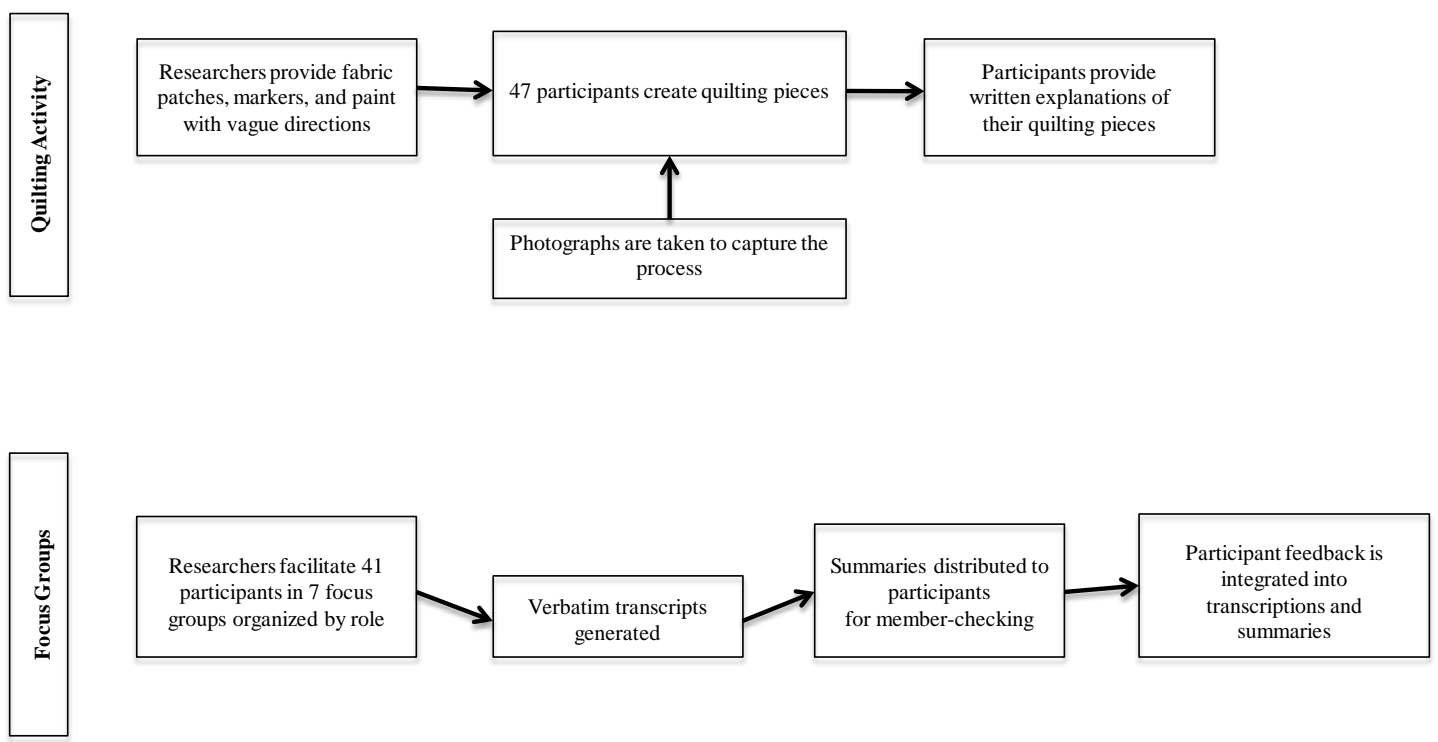
Figure 2

Summary of thematic analysis framework
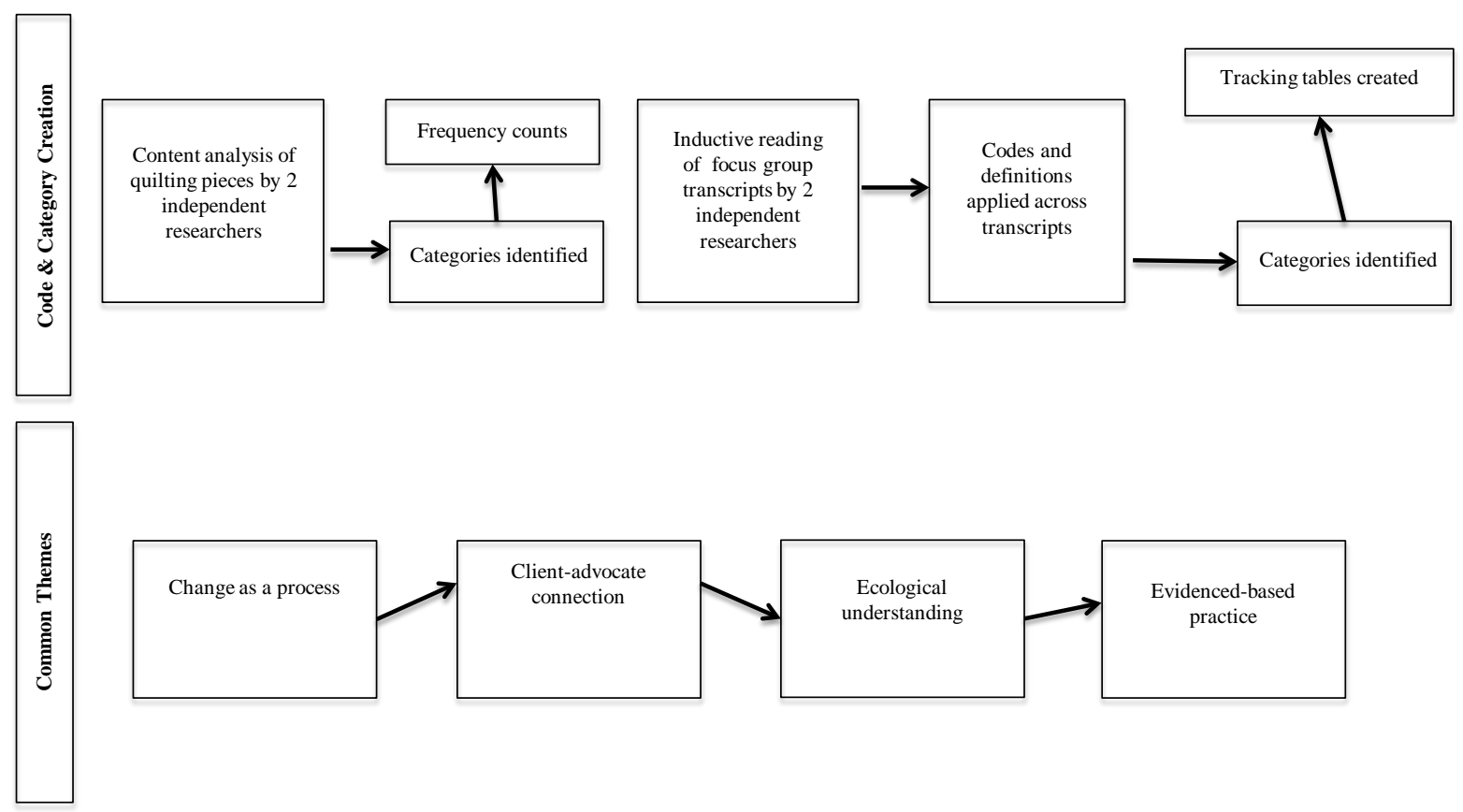

Figure 3

Categories of quilting activity

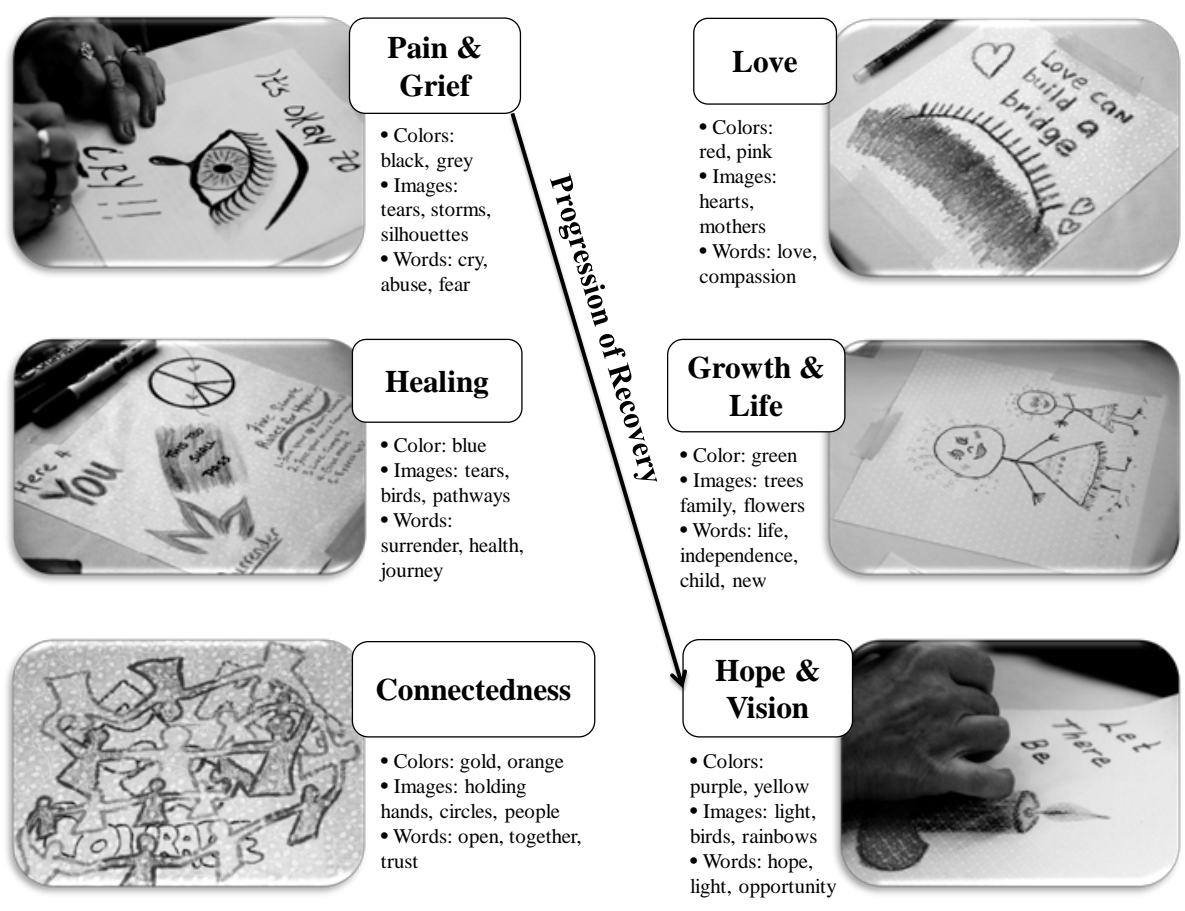




\section{Results}

Four themes revealing participants' emotion, candor, and passion as well as their commitment to the care and advocacy of women and children affected by FASD were generated: change as a process, client-advocate connection, ecological understanding, and evidence-based practice.

\section{Change as a Process}

Many of the quilting pieces captured the change progression associated with client recovery as a process rather than a single event, while focus group participants discussed changes in program delivery over time. The round shapes, cursive letters, and fluid images (e.g., paths, water) common to many quilting pieces suggest that service delivery personnel have a mindful view of how relationship-building is developmental. The inclusion of images such as eye, tear, hand, heart, tree, sun and rainbow suggest a movement through recovery-acknowledging pain and envisioning a better and possible future. This idea is further triangulated by illustrations, quotes, words, and colors related to different aspects of the recovery process, moving from pain and grief, to healing, to growth and new life. This is not surprising, as the complex and chaotic lives (which may involve history of abuse, legal problems, mental health issues, and poverty) of vulnerable women and children leave these clients at different stages of readiness for change.

The majority of focus group participants reported tremendous growth in their field, fueled by increased government involvement and the emergence of new program sites in various locations across Alberta. These changes have led to a greater number of individuals with FASDs being able to access and receive services, as well as an increase in public awareness campaigns. As one participant explained, "Within the program itself, I think . . . the biggest change is working with that exponential growth. I've been with [PCAP] for two years and we've gone from four clients to fifty-two..." Despite the challenges of working with this specialized population, an overall optimism was apparent, deriving from participants' belief in the importance of their work and their clients' potential for positive change and growth.

\section{Client-Advocate Connection}

Across focus group discussions and quilting pieces, the connection between clients and advocates was described as paramount to the success of treatment and prevention services. Among the major strengths of PCAP is its focus on relationship building, in terms of approaches for working with clients, reflective supervision, supporting colleagues, and collaborating with other service providers. In the quilting illustrations, connectedness was suggested through images of holding hands, pathways, circles, and people in orange and gold coloring. Similarly, focus group participants emphasized the flexibility of the PCAP model and its emphasis on non-judgment as crucial when working with special populations, and key to the establishment of the client's trust in the process of recovery and the person lending support. As one participant explained: “... programs and strategies don't heal people, relationships do and that's just so important . . . It's not the programs that are great programs, it's the individuals who are [leading them]." The importance of trust was shown in the quilting pieces through depictions of closeness between people; the inclusion of hearts, interlocking hands and overlapping figures; and the use of words like "together," "trust," and "love." In focus groups, participants reported that their work was directed at increasing client self-efficacy and that a woman's sense of connectedness to others is central to her growth, development, and definition of self. Rather than adopting a me-you perspective, PCAP advocates strive to create a mindset of we, believing that women with drug and/or alcohol addiction should never feel isolated in their journey of recovery. As one advocate expressed in the focus group sessions:

Nobody ever gave them a chance. Now, they finally have somebody to support them to actually succeed and move forward in their lives instead of being stuck in . . . their addictions and having babies and losing them. [It can be a] vicious cycle that they get stuck in. So just having somebody there to help them [get] unstuck, [that is what's important] ...

\section{Ecological Understanding}

The need for improved understanding of individuals with FASD and the advocates who work with them across services and contexts emerged as a common theme. The quilting pieces revealed stories of pain and grief, but also of overcoming and hope. Several focus group participants commented on the therapeutic aspect of the exercise, saying that they "couldn't have guessed how [their] pieces would have turned out," or that the feelings and thoughts expressed had been buried deep inside. This suggests a need for increased reflection on how our work is affecting our clients and ourselves, which demonstrates the usefulness of creative outlets that allow us to tell a story. It also shows how cathartic and exploratory such exercises can be. Although the quilting pieces revealed hopefulness, connectedness, compassion, and strength-demonstrating the participants' passion, dedication, and belief that their work can and does make a difference-the desire for meaning in activity appears to be central to their request for continued professional learning and personal growth. The quilting pieces provided evidence of the humanistic and relationship-based approaches of PCAP, illustrating acceptance and value of the client and the human, and the reality of our need for caring communities. This sentiment was corroborated in focus group sessions; as one participant stated, "There are no throwaway people." This highlights the need for us to do our best to help our clients become the most capable women and mothers they can be. No matter the struggle, each person is valuable and has the capacity for positive change.

\section{Evidence-Based Practice}

The majority of focus group participants reported that their prevention programs were strongly research-based, yet there was little reference to the influence of research in the 
quilting pieces. The same participants also highlighted the fact that PCAP is continuously evaluated, which helps inform prevention activities and affords advocates confidence in the effectiveness of their services. As one participant commented, "[Research-based information on] PCAP not only provides credibility to the work that you're doing, but it gives you that focus in how you're going to move forward." Yet, they were conflicted in their recognition of the need for evidence-based practice. Although they saw research and best practice as useful, they also stressed that accessing current information on such research is incredibly time-consuming. However, several workers stated that they currently receive FASD research by e-mail through their agencies, and were enthusiastic about the potential of a new avenue for research access. As one advocate expressed: “...it's exciting . . . to look forward to having . . . more research information that can be used ... [A] database would be awesome [in helping with access]...”

Another key idea emerging from focus group discussions on how to ameliorate research access was to increase links between FASD researchers and frontline workers. For participants who had this contact, the experience had been largely positive: "It was very good . . . working with [Jessica], who ... does a lot of workshops around FASD. She's my mentor, and so it really helps to have someone who is that knowledgeable around FASD to be your support and in your corner..." Indeed, increased opportunity to network with researchers was delineated as critical to the improved use of research, as was establishing evaluations for programs to increase evidence-based practices in the field.

\section{Discussion}

Just as the metaphor of the quilt emphasizes the personal stories within a collective work, so too can research be used to provide connections between different methods through data integration (Koelsch, 2008). Whether visual or oral, information about participants' experiences is gleaned and made available for comparative analysis to establish key elements of FASD prevention and service delivery, and to identify where strengths and challenges lie in working toward the best possible outcomes. The integration of focus groups with a quilting activity provided an appropriate and trustworthy means of accessing the prevention specialists' professional and personal experiences, which have important practice and methodological implications. While the integrated findings of this study have the strong potential for informing FASD policy, strategic planning, and programming, the account of the study's innovative methodological approach provides a real-life example of its usefulness within this type of research context. In a time when research is increasingly expected to show its relevance to policy and social change, images have the ability to reach a wide audience, breaking through common resistance and engaging viewers in a new way of seeing FASD (Weber, 2008). Sharing the quilt with government officials involved in the FASD-CMC may provoke critical questions about the value of FASD services and spark individual and collective action toward superior strategic planning and programming in this area (Deshpande et al., 2005). It is also plausible that emotional engagement created through visual research data may push policymakers to listen to advocates' concerns and suggestions more readily, providing a different framework for the health, justice, social, and educational issues surrounding FASD.

The findings from this study contribute improved understanding of the significance of relationships, reflection, and research in the work of FASD prevention specialists. It is through our interactions with others and our diverse experiences that we truly learn about ourselves; thus, a research approach that incorporates reflection, subjective awareness, connection, communication, and creativity can provide significant value to a project and the participants involved (Wright, personal communication, 2011). This idea is also wholly consistent with the PCAP model, which invites openness and candidness from clients, advocates, and supervisors alike (Ernst et al., 1999) and encourages learning through reflection on past and present experiences and taking an honest look at oneself.

The novel inclusion of a quilting activity along with focus groups also allowed for reciprocity in teaching and learning between researchers and participants, giving rise to enhanced ecological understanding (Job et al., 2012). As O’Sullivan and Taylor (2004) describe, “[Knowledge is] not individually derived and held but . . . generated in relationships with others" (p. 21). It is important to note the transformational effect that involvement in this multimethod study had on research team members, many of whom had no prior knowledge of or involvement with the FASD world. As one doctoral student relayed, "In our research, we are usually focused on the child, their deficits, and the interventions needed ... This focus on prevention and the struggles that are involved in working with this population definitely added to my understanding." Added to the participant summaries was a supplement of reflections from researchers, detailing how collecting the data had personally impacted them. This provided further value to participants, who believed their input normally fell on deaf ears, in that they were able to read about the effect of their words and illustrations on the researchers. It also encouraged researchers to reflect on their involvement and the importance of making an authentic connection with participants in community-based research (Job et al., 2012).

With respect to research, participants expressed a fervent desire for FASD-related research and program evaluation to further evidence-based practice, a need which is consistent with the literature (Baugh et al., 2011). Participants also suggested a greater push to work with other agencies to bring FASD to the forefront of public awareness and increase accessibility to community services, as best practice means advocates cannot work in isolation (Brown, 2004). This is noteworthy, and ties in well with the argument for a multi-method approach to data collection. If a primary goal in FASD strategic planning is to enhance community awareness (McCarty, Waterman, Burge, \& Edelstein, 1999), it only makes sense to 
incorporate ways to increase understanding and accessibility of information into research methods. PCAP advocates recommended the creation of an online database to enhance information sharing and communication between FASD researchers, frontline workers, provincial networks, and community agencies. Access to consistent and timely reporting of program outcomes has the potential to increase collaboration between frontline workers across communities and settings (Pei \& Poth, 2009). Additionally, the usefulness of the quilt lies in its visual presentation of information, telling individual stories and enabling community partners to see how the parts make up the whole (Weber, 2008), helping them to contextualize the material they read and see the real people behind it.

\section{Limitations}

The current study is limited by the methods used for sampling, data collection, and analysis. The first is related to the study's use of convenience sampling; that is, the sample consisted of those attending the Alberta PCAP Council Annual General meeting who were willing to participate. It might be important for a follow-up study to confirm findings across the entire PCAP population. The second is related to the study's use of focus groups and a quilting activity; the data from the focus groups is limited to what participants were willing to share in a small-group format, and information from the quilting activity is limited to what participants were willing to express visually. The third is related to the study's use of thematic content analysis and constant comparison. While these approaches are inherent in grounded theory methodology, thematic interpretation has been used in tandem with a constant comparison approach, which has been described as "taking one piece of data (one interview, one statement, one theme) and comparing it with all others that may be similar or different in order to develop conceptualizations of the possible relations between various pieces of data" (Thorne, 2000, p. 2). Given the interpretative experience of quilting, measures were undertaken to mitigate researcher bias (i.e., member checking, multiple coders) to accommodate the two different levels of analysis in the project. Further research is needed to (a) address the limitations highlighted within the findings, as well as to (b) replicate this study for greater generalization and understanding of the PCAP experience.

\section{Conclusions}

Visual methods should be considered in research, as they add a personal element to a traditionally impersonal process. What makes them unique is that they represent an effort to understand a participant's lived experience, recognizing the wisdom that comes from consideration of the whole self-head, heart, and body (Job, O’Riordan, \& Taylor, 2012). To this end, it is argued that creative expression through quilting holds promise as an avenue for making sense of and expressing experience, especially among those whose work involves emotional labor (Hochschild, 1983) (e.g., nurses, doctors, flight attendants), and for whom emotional expression may be undesirable in the confines of the workplace. In the present study, the use of focus groups with quilting allowed us greater insight into what it means to work as a prevention specialist in the field of FASD and how this work plays into the emotional and relational self. As well, the combination of methods helped to generate a comprehensive and shared understanding related to best practices in relationships, reflection, and research, with the goal of affecting FASD policy, strategic planning, and programming. There is still much work to be done; however, seeing the participant value of a multimethod approach and potential for increased dissemination and influence gives us hope for positive change, more nuanced knowledge claims, and an improved framework for research in the field.

\section{Acknowledgements}

This research would not have been possible without support from the following collaborators:

- The Alberta Community-based Collaboration and Evaluation Research Team (ACCERT) for project conceptualization, data collection, analysis, and reporting;

- Lin Taylor, Master Quilter;

- Bev Ewan for creative and technical support and masterful quilting;

- Bev Edwards-Sawatzky for creative support;

- The Alberta PCAP Council for promoting opportunities for innovation; and

- The men and women of PCAP whose dedication to FASD prevention is both noteworthy and inspiring.

We are truly appreciative.

\section{References}

Ausband, L. (2006). Qualitative research and quilting: Advice for novice researchers. The Qualitative Report, $11,764-770$.

Banks, M. (2001). Visual methods in social research. London, England: Sage Publications.

Baugh, L., Denys, K., Hutchison, M., Kully-Martens, K., Tamana, S., \& LaFrance, M. A. (2011, November). Translating research into practice. Paper presented at the 2011 Alberta FASD Conference, Calgary, AB.

Brown, J. D. (2004). Family supports for children who have alcohol-related disabilities. Developmental Disabilities Bulletin, 32, 44-61.

Burd, L., Klug, M. G., Martsolf, J. T., \& Kerbeshian, J. (2003). Fetal Alcohol Syndrome: Neuropsychiatric phenomics. Neurotoxicology \& Teratology, 25, 697705.

Chudley, A. E., Conry, J., Cook, J. L., Loock, C., Rosales, T., \& LeBlanc, N. (2005). Fetal Alcohol Spectrum Disorder: Canadian guidelines for diagnosis. Canadian Medical Association Journal, 172, 1-21.

Connor, P., \& Streissguth, A. (1996). Effects of prenatal exposure to alcohol across the lifespan. Alcohol Health Research, 20, 148-157. 
Creswell, J. W. (2008). Research design: Qualitative, quantitative, and mixed methods approaches (3rd ed.). Thousand Oaks, CA, United States: Sage Publications.

Deshpande, S., Basil, M., Basford, L, Thorpe, K., PiquetteTomei, N., Droessler, J., Cardwell, K., Williams, R. J., \& Bureau, A. (2005). Promoting abstinence among women: Potential social change strategies. Health Marketing Strategy, 23, 45-67.

Eisner, E. (1995). What artistically crafted research can help us to understand about schools. Educational Theory, 45, 1-6.

Emmel, N., \& Clark, A. (2011). Learning to use visual methodologies in our research: A dialogue between two researchers. Forum Qualitative Sozialforschung /Forum: Qualitative Social Research, 12, Art. 36.

Ernst, C. C., Grant, T. M., Streissguth, A. P., \& Sampson, P. D. (1999). Intervention with high-risk alcohol and drug-abusing mothers: II. Three-year findings from the Seattle model of paraprofessional advocacy. Journal of Community Psychology, 27, 19-38.

Galman, S. A. C. (2009). The truthful messenger: Visual methods and representation in qualitative research in education. Qualitative Research, 9, 197-217.

Gould, L., Job, J., St. Arnault, D., Pei, J., \& Poth, C. (2012). Shaping the future of Fetal Alcohol Spectrum Disorder (FASD): Policy, practice, and research in Alberta. Poster presented at the 5th Annual Educational Psychology Graduate Student Colloquium, Edmonton, $\mathrm{AB}$.

Government of Alberta. (2008). FASD 10-year strategic plan. Retrieved from www.fasd-cmc.alberta.ca/ uploads/1004/fasd10yrplanfinal86321.pdf

Grant, T., Ernst, C. C., Pagalilauan G., \& Streissguth, A. P. (2003). Post-program follow-up effects of paraprofessional intervention with high-risk women who abused alcohol and drugs during pregnancy. Journal of Community Psychology, 31, 211-222.

Grant, T. M., Ernst, C. C., \& Streissguth, A. P. (1996). An intervention with high-risk mothers who abuse alcohol and drugs: The Seattle Advocacy Model. American Journal of Public Health, 86, 1816-1817.

Grant, T. M., Ernst, C. C., \& Streissguth, A. P. (1999). Intervention with high-risk alcohol and drug-abusing mothers: I. Administrative strategies of the Seattle model of paraprofessional advocacy. Journal of Community Psychology, 27, 1-18.

Grant, T. M., Ernst, C. C., Streissguth, A. P., Phipps, P., \& Gendler, B. (1996). When case management isn't enough: A model of paraprofessional advocacy for drug- and alcohol-abusing mothers. Journal of Case Management, 5, 3-11.

Grant, T. M., Streissguth, A. P., \& Ernst, C. C. (2002). Intervention with alcohol- and drug-abusing mothers and their children: The role of the paraprofessional. The Source: Newsletter of the National Abandoned Infants Assistance Resource Center, 11, 5-26.

Health Council of Canada. (2011). Alberta FASD CrossMinistry Committee (FASD-CMC). Retrieved on March 3, 2013 from http://innovation.healthcouncilcanada.ca/innovationpractice/alberta-fasd-cross-ministry-committee\#
Hochschild, A. R. (1983). The managed heart: Commercialization of human feeling. Berkeley, CA, United States: University of California Press

Job, J., O’Riordan, T., \& Taylor, L. (2012). Capturing the multifaceted and complex nature of work with individuals with FASDs: Practical guidelines for weaving quilting into community-based research. Oral presentation at the Second European Conference on FASD, Barcelona, Spain.

Kelly, S. J., Day, N., \& Streissguth, A. P. (2000). Effects of prenatal alcohol exposure on social behaviour in humans and other species. Neurotoxicology and Teratology, 22, 143-149.

Koelsch, L. E. (2008). Unlabeled sexual experiences: Quilting stories and reenvisioning discourses. (Doctoral dissertation). Miami University, Miami, Florida, United States.

Leonardson, G. R., \& Loudenburg, R. (2003). Risk factors for alcohol use during pregnancy in a multistate area. Neurotoxicology \& Teratology, 25, 651-658.

Lincoln, Y. S., \& Guba, E. G. (1985). Naturalistic inquiry. Beverly Hills, CA, United States: Sage Publications.

McCarty, C., Waterman, J., Burge, D., \& Edelstein, S. B. (1999). Experiences, concerns, and service needs of families adopting children with prenatal substance exposure: Summary and recommendations. Child Welfare, 78, 561-577.

O’Sullivan, E., \& Taylor, M. (2004) Learning towards an ecological consciousness. New York, NY, United States: Palgrave Macmillan.

Pei, J., Denys, K., Hughes, J., \& Rasmussen, C. (2011). Mental health issues in fetal alcohol spectrum disorder. Journal of Mental Health, 20, 438-448.

Pei, J., \& Poth, C. (2009). Effective strategies for bridging the communication gap in service delivery for FASD individuals. Presented at the 2009 Alberta FASD Conference, Edmonton, AB.

Pink, S. (2007). Doing visual ethnography. London, England: Sage.

Poth, C., \& Pei, J. (2012). Fetal alcohol spectrum disorder (FASD) 10-year strategic plan: A summary of the Year Five evaluation project. Unpublished manuscript. University of Alberta, Edmonton, Alberta.

Rasmussen, C. (2005). Executive functioning and working memory in Fetal Alcohol Spectrum Disorder. Alcoholism: Clinical \& Experimental Research, 29, 1359-1367.

Rose, G. (2001). Visual methodologies: A guide to the interpretation of visual materials. London, England: Sage Publications.

Sampson, P. D., Streissguth, A. P., Bookstein, F. L., Little, R. E., Clarren, S. K., Dehaene, P., \& Graham Jr., J. H. (1997). Incidence of fetal alcohol syndrome and prevalence of alcohol-related neurodevelopmental disorder. Teratology, 56, 317-326.

Savenye, W. C., \& Robinson, R. S. (2003). Qualitative research issues and methods: An introduction for educational technologists. In D. H. Jonassen (Ed.), Handbook of research on educational communications and technology (2nd ed., pp. 1045-1071). Retrieved from http://www.aect.org/edtech/39.pdf 
Steinhausen, H. C., \& Spohr, H. L. (1998). Long-term outcome of children with fetal alcohol syndrome: Psychopathology, behavior, and intelligence. Alcoholism: Clinical \& Experimental Research, 22, 334-338.

Streissguth, A. P., Barr, H. M., Kogan, J., \& Bookstein, F. L. (1996). Understanding the occurrence of secondary disabilities in clients with fetal alcohol syndrome (FAS) and fetal alcohol effects (FAE): Final report to the Centers for Disease Control and Prevention. Seattle, WA, United States: University of Washington, Fetal Alcohol and Drug Unit.

Streissguth, A. P., Sampson, P. D., Olson, H. C., Bookstein, F. L., Barr, H. M., Scott, M., Feldman, J., \& Mirsky, A. F. (1994). Maternal drinking during pregnancy: Attention and short-term memory in 14year-old offspring-A longitudinal prospective study. Alcoholism: Clinical \& Experimental Research, 18, 202-218.

Thorne, S. (2000). Data analysis in qualitative research. Evidence Based Nursing, 31, 68-70.

Weber, S. (2008). Chapter 5: Using visual images in research. In J. G. Knowles \& A. L. Cole (Eds.), Handbook of the arts in qualitative research: Perspectives, methodologies, examples, and issues (pp. 41-54). London, England: Sage Press. 